\title{
Effect of exogenously applied kinetin and glycinebetaine on metabolic and yield attributes of rice (Oryza sativa L.) under drought stress
}

\author{
Jalal-ud-Din $^{1 *}$, Sami Ullah Khan ${ }^{2}$, Ahmad Khan $^{3}$ and Shahzad Naveed ${ }^{4}$ \\ ${ }^{I}$ Wheat Wide Crosses Program, PGRI, NARC, Islamabad, Pakistan \\ ${ }^{2}$ Department of Agricultural Sciences, University of Haripur, Khyber Pukhtunkhwa, Pakistan \\ ${ }^{3}$ Land Resources Research Institute, NARC, Islamabad, Pakistan \\ ${ }^{4}$ Islamabad College for Boys $G$ 6/2, Pakistan
}

\begin{abstract}
Drought is one of the major abiotic stress responsible for yield reduction of crops worldwide. Kinetin (KIN) and glycine betain (GB) have an essential role in regulating cell division during the early phase of grain setting; assimilate flow and osmotic adjustment under drought stress. Effects of exogenously applied kinetin and glycine betain on some metabolic and yield attributes of two rice (Oryza sativa L.) cultivars viz., Basmati-385 and KS-282 were evaluated under water stress. KIN and GB were applied by spraying the plants at flowering stage before drought stress treatment. Both treatments significantly increased proline concentration in leaves and panicles of both the rice cultivars as compared to their control. They also increased soluble sugar in the leaves and panicles under water stress. Starch concentration in leaves and panicles of both the rice cultivars were increased significantly $(\mathrm{p}<0.05)$ by KIN and GB spray. However, the magnitude of increase of the starch in panicles was more pronounced in cv. Basmatti-385 than cv. KS-282. Under the applied water stress both KIN and GB spray significantly increased paddy yield in both the rice cultivars. Both KIN and GB spray significantly increased the number of seeds per panicle in both the rice cultivars. The quantitative increase in paddy yield and seed number was greater in cv. Basmatti-385 as compared to cv. KS-282. Exogenous application of KIN at flowering stage could be used as an effective treatment to mimic the adverse effects of water stress in fine rice cultivars.
\end{abstract}

Key words: Kinetin, Drought, Glycine betain, Proline, Starch, Sugar, Rice, Yield

\section{Introduction}

Rice is one of the most essential cereals and biggest irrigated crop in the world. Rice crop is a major user of fresh water and availability of water for irrigation is increasingly getting scarce (Laborte et al., 2012). Therefore, drought tolerance improvements are important if the efficiency of food production is to be increased. It is estimated that $50 \%$ of the world rice production is affected more or less by drought (Bouman et al., 2005).

Plant cells undergo numerous metabolic changes in response to water deficit, some of which

Received 28 February 2014; Revised 02 April, 2014; Accepted 19 May 2014; Published Online 15 December 2014

*Corresponding Author

Jalal-ud-Din

Program Leader,

Wheat Wide Crosses Program, PGRI, NARC, 45500-

Islamabad, Pakistan

Email: jalaludin10@yahoo.co.uk may have adaptive significance (Cha-um et al., 2010). These responses may cause alteration of the whole metabolic pathway leading to an accumulation or depletion of metabolites such as carbohydrates, organic acids, amino acids, quaternary ammonium compounds, hormones and stress related increases in the activity of a number of hydrolytic enzymes. The organic compounds, referred as compatible solutes such as proline, soluble sugars etc maintain the cellular osmotic potential/ osmoregulation and can be used as a metabolic marker in relation to drought stress (Thapa et al., 2011).

Drought is one of the most important abiotic stresses that severely reduce crop productivity. Plant growth regulators and osmoprotectant have been found to play a central role in the integration of the responses expressed by plants under stress conditions. Glycine betaine (GB) a member of quaternary ammonium compounds has been found to act as osmoprotectant which improves the growth and development of crop plants. It protect 
photosynthetic machinery and stabilize the structure of Rubisco under drought (Ashraf and Foolad, 2007; Chen and Murata, 2008; Rezaei et al., 2012). GB is an efficient protective agent by stabilizing the structures and functions of certain macromolecules. Previously research has shown that exogenous GB application has potential to sustain and enhance the growth and yield in monocot crops such as rice (Farooq et al., 2008), and wheat (Ma et al., 2007). GB foliar spray has been shown to improve growth performances of fine grain aromatic rice seedlings subjected to drought stress (Farooq et al., 2008, 2010).

In rice reproductive stage is most sensitive to drought in rice where reduction in anthesis occurs as the panicle water potential decreases and results in large scale spikelet sterility. Drought stress during flowering can interrupt floret initiation, translocation of assimilates to the grain which lowers grain filling and size (Akramet al., 2013; Ahadiyat et al., 2014). Plant growth hormone regulates cell division, elongation and influence buds formation in plants. It direct assimilates flow, enhances nutrient absorption and translocation toward the site of action (Davies, 2010). Kinetin is involved in active cell growth during the early stage of grain formation. In cereals, grain yield depend upon the plant source / sink association. Rice is significantly reliant on the source leaves for grains formation as photosynthesis in the emerging panicles contributes only less to grain formation. Changes in hormones play main role in source/ sink variations, and kinetin seem to be a major factor affecting these changes (Radchuk et al., 2012). Therefore, hormone triggered consolidation of source/-sink might be a significant factor for the evolution of water stress tolerant rice. In this study an attempt was made to assessing the role of KIN and GB in drought tolerance improvement and yield of rice. The levels of osmoprotectants and thus stress tolerance could be increased by breeding if the plants have the capacity to synthesize them, if not these could be introduced by genetic engineering or simply by external application. External application is relatively cheaper and quicker way of assessing the role of osmoprotectants in rice cold stress tolerance.

Keeping in view these facts a research study was undertaken to analyze the effect of exogenously applied kinetin and glycine betaine in rice under water stress conditions to mitigate the effect of water stress.

\section{Materials and Methods \\ Plant material}

The experiment was conducted during JuneSeptember in a glasshouse under controlled temperature between $25-38 \pm 2^{\circ} \mathrm{C}$. Sterilized seeds of two local rice cultivars (one coarse and one fine) viz., KS-282 and Basmati-385 were germinated and seedlings were grown in a sand culture. Twenty days old rice seedlings were transplanted into pots (30 x $40 \mathrm{~cm}$ size) containing $10 \mathrm{~kg}$ soil. The pots were filled with clay soil from rice field area, National Agricultural Research Center, Islamabad, Pakistan. The soil was air dried and sieved through $5 \mathrm{~mm}$ sieve before filling in the pots. Recommended fertilizer mixture was applied to the pots at $125,95,65,5$ and $1 \mathrm{~kg} \mathrm{ha}^{-1} \mathrm{~N}^{3}-\mathrm{P}_{2} \mathrm{O}_{5}$ and $\mathrm{K}^{+}, \mathrm{Zn}^{+2}$ and $\mathrm{B}^{3+}$ on soil weight basis in the form of urea, di-ammonium phosphate, sulphate of potash, zinc sulphate and borax. All the $\mathrm{P}, \mathrm{K}$, zinc and boron were applied at the time of sowing while $\mathrm{N}$ was applied in three equal splits. The pots were arranged in randomized complete block design.

\section{Hormonal and drought treatment}

At the end of booting stage and panicle initiation growth stage (end of booting stage, 50 milliliter aqueous solution of glycine betaine (100 $\mathrm{mM})$ and kinetin $(1 \mathrm{mM})$ were applied as a foliar spray three times per week while control plants were sprayed with distilled water. Three days after hormonal treatments, drought stress was imposed in plants by withholding irrigation for 5-7 days till the signs of temporary wilting/leaf rolling appeared. The estimated soil moisture content during water withholding time was $14 \%$. The stress duration was from panicle initiation till soft dough stage of grains. Immediately after drought stress treatment, samples of flag leaves and panicles were collected and pots were irrigated.

The leaf and panicle sample were analyzed for estimation of proline, soluble sugar and starch contents as described in IRRI, (1976).

Leaf proline was analyzed using the method of Bates et al. (1973). Fresh plant tissue was extracted with 3\% aqueous 5-sulfosalicylic acid and the filtrate was reacted with freshly prepared ninhydrin solution at $100^{\circ} \mathrm{C}$ for 1 hour. The reaction mixture was extracted with 4 milliliter toluene and the absorbance of the chromophore containing toluene was recorded at $520 \mathrm{~nm}$. Leaf tissues were homogenized in $10 \mathrm{ml}$ of $80 \%$ (v/v) ethanol and the mixture was boiled for $10 \mathrm{~min}$ for soluble sugar estimation. The samples were centrifuged at $3000 \mathrm{~g}$ for $10 \mathrm{~min}$ and the supernatant discarded. Another extraction was done using $10 \mathrm{ml}$ of hot $80 \%$ 
ethanol. The supernatant portions were pooled and soluble sugars were determined by the phenol sulfuric acid method (Dubois et al., 1956) using glucose as standard. Starch was estimated from the dried residue left in the centrifuge tubes after sugar extraction. Two milliliter of distilled water was added to the centrifuge tubes containing the dried residue and boiled in water bath for 15 minutes. The tubes were allowed to cool and $2 \mathrm{ml} 9.2 \mathrm{~N}$ $\mathrm{HClO} 4 \mathrm{added}$ while stirring continuously. Volume was made up to about 10-ml and centrifuged. This suspension was stirred for 15 minutes and made up to $10-\mathrm{ml}$ with distilled water. This was centrifuged, supernatant combined and volume made up to 50$\mathrm{ml}$ with distilled water. The starch was analysed from this extract. Then 5-ml of anthrone reagent was added to 2-ml of the starch extract. The solution was boiled for 10 minutes and absorbance was measured at $620 \mathrm{~nm}$ on spectrophotometer after cooling.

At physiological maturity, panicles from individual plants were harvested and oven-dried at $80^{\circ} \mathrm{C}$ for $72 \mathrm{~h}$. Theagronomic data regarding number of grains per panicle was recorded and total paddy yield (weight) was recorded.

The collected data were subjected to analysis of variance using statistical package Satistix 8.1. The differences among the treatment means were done using the Tukey test at $5 \%$ significance level.

\section{Results \\ Proline content}

The data (Figure 1) revealed significant variation among control (without KIN and GB spray and water stress conditions), kinetin and glycine betaine spray. The higher proline accumulation in leaves (Figure 1a) occurred by KIN (26.9\%) and GB spray in KS-282 with respect to its control. However, proline accumulation was statistically equal under KIN and GB spray in KS282. Basmati-385 accumulated (40.9\%) increase in proline content as compared to KS-282 by GB application under water stress conditions. Similarly, there was significant (Figure 1b) increase in panicles proline accumulation of both the rice cultivars with respect to their untreated control. The quantitative increase in panicle proline was greater by KIN spray in both the rice cultivars. The magnitude of increase in proline was greater (about $29.2 \%$ ) in KS-282 under water stress conditions.

\section{Sugar content}

The data (Figure 2a) depicted significant differences for sugar accumulation in leaves between two rice cultivars by KIN and GB spray.
Significant increase $(27.7 \%)$ in soluble sugar was observed in leaves of KS-282 with respect to its untreated control under water stress conditions. Similarly, there was significant (Figure 2b) increase in sugar accumulation in panicles of both the rice cultivars with respect to their untreated control. Both KIN and GB spray were found to significantly increase soluble sugar in panicles of both the rice cultivars under water stress conditions.
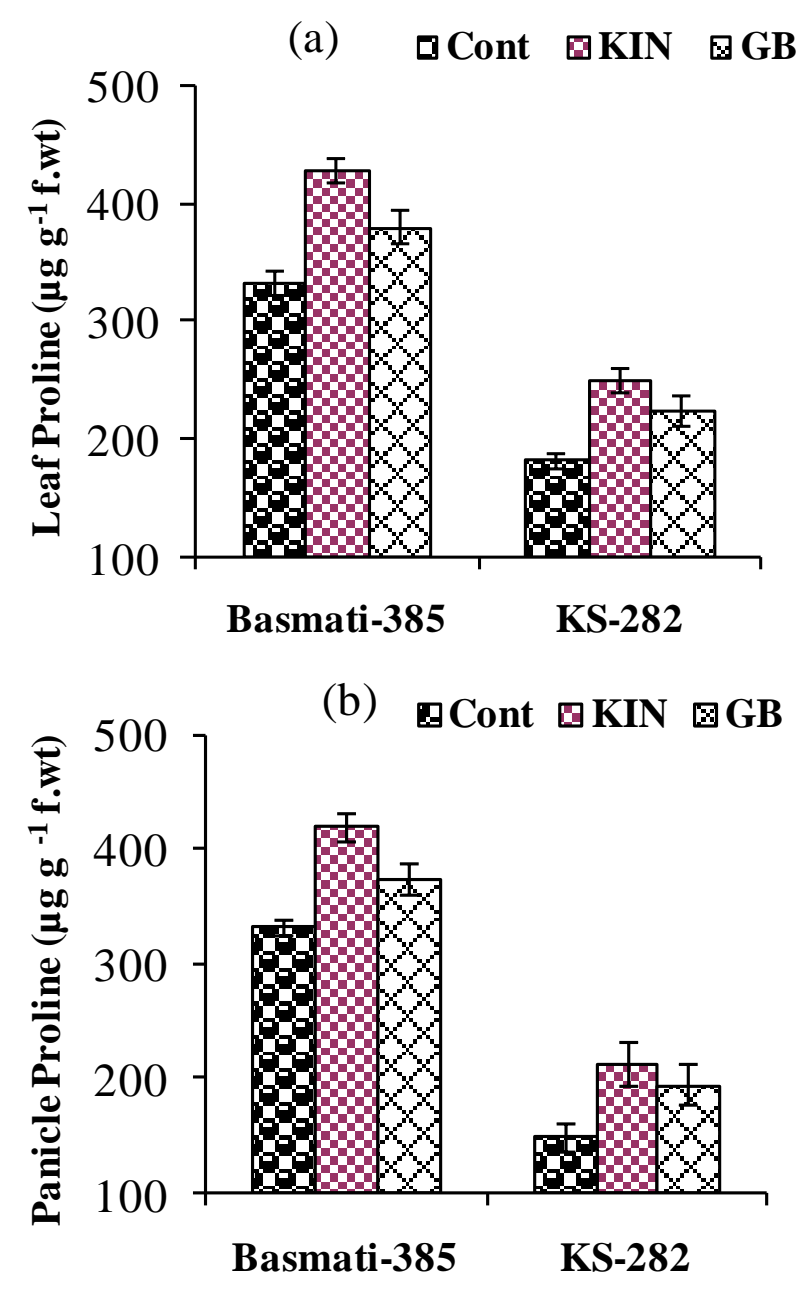

Figure1. Effect of Glycinebetaine $(100 \mathrm{mM})$ and Kinetin $(1 \mathrm{mM})$ on the leaf (a) and panicle (b) proline concentration ( $\mu \mathrm{g} \mathrm{g}-1$ fresh weight) of riceunder drought stress. The vertical bars indicate standard error (SE) of mean ( $\mathrm{n}=3$ ). Cont-Control (distilled water spray), KINKinetin, GB-Glycinebetaine.

\section{Starch contents}

Significant variation of leaf starch content was found in both the rice cultivars with regard to their respective control (Figure 3a). The magnitude of increase in leaf starch was relatively higher (59.6\%) in Basmati-385 by KIN spray at panicle initiation stage under water stress conditions. Both KIN and 
GB significantly (Figure 3b) increased panicle starch of both the rice cultivars under water stress conditions. KIN and GB spray caused $75.3 \%$ and $65.7 \%$ increase in panicle starch of Basmat-385 under water stress conditions respectively. However, there was non-significant difference for panicle starch content between the two rice cultivars.

(a)

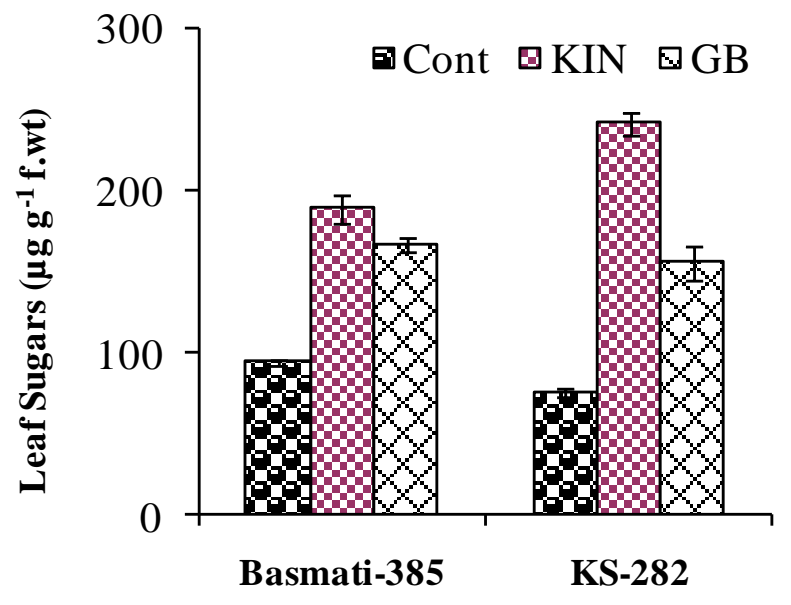

(b)

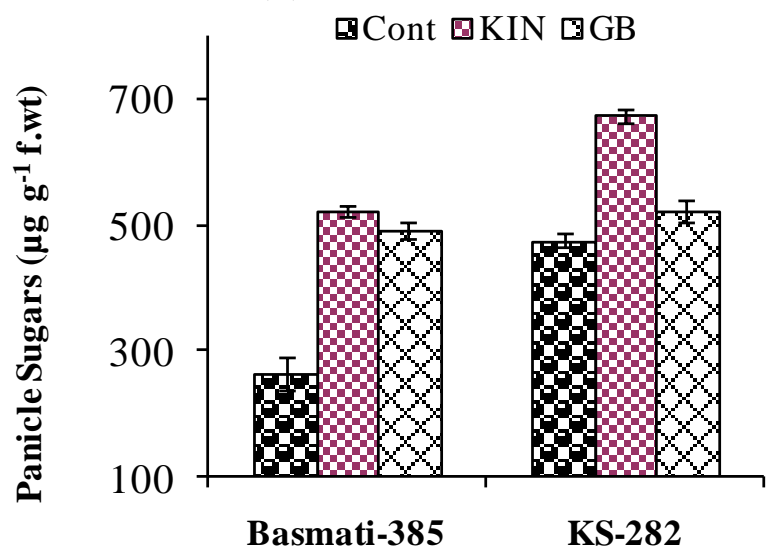

Figure 2. Effect of Glycine betaine $(100 \mathrm{mM})$ and Kinetin $(1 \mathrm{mM})$ on the leaf (a) and panicle(b) sugar concentration $\left(\mu \mathrm{g} \mathrm{g}^{-1}\right.$ fresh weight) of rice under drought stress. The vertical bars indicate standard error (SE) of mean $(n=3)$.Cont-Control (distilled water spray), KINKinetin, GB-Glycine betaine.

\section{Paddy Yield Plant}

The data regarding the Paddy wt. of the two rice cultivars cv. Basmatti-385 and KS-282 is presented in the Table 1. The analysis of data revealed that the paddy wt. in both the rice cultivars were increased significantly statistically $(\mathrm{p}=0.05)$ as compared to their control under drought condition. The data showed significant variation for paddy yield of both the rice cultivars under various treatments. Significant increase in paddy yield was noted in Basmati-385 by
KIN spray with respect to its untreated control However, GB spray caused (31.8\%) significant increase in paddy yield of KS-282 with respect to its control. Nonetheless the amount of increase in paddy yield of Basmai-385 was relatively greater (49.6\%) through KIN than GB spray under water stress conditions. The data regarding the seeds per panicle of two rice cultivars cv. Basmatti-385 and KS-282 is shown in the Table 1 . The analysis of data revealed that the seeds per panicle in both the rice cultivars were increased significantly statistically $(p=0.05)$ with KIN than GB spray under water stress conditions as compared to their control.

\section{Discussion}

Under water stresses, Glycine betaine and Cytokinin foliar application increased the leaf and panicle proline, soluble sugar and starch in the leaves and panicles of both the rice cultivars as compared to their control.

(a)

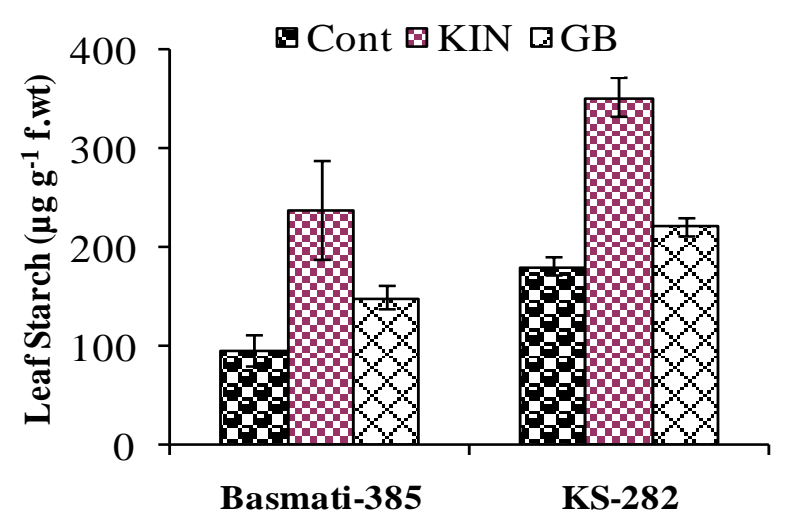

(b)

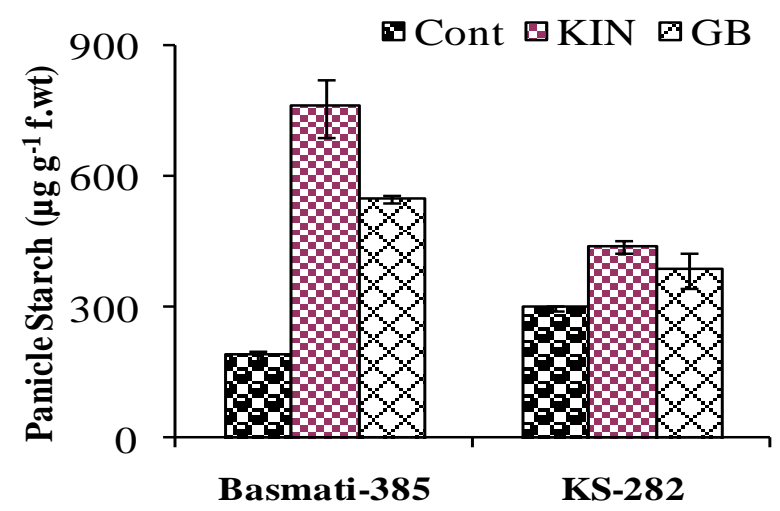

Figure 3. Effect of Glycine betaine $(100 \mathrm{mM})$ and Kinetin (1 mM) on the leaf (a) and panicle(b) starch concentration $\left(\mu \mathrm{g} \mathrm{g}^{-1}\right.$ fresh weight) of rice under drought stress. The vertical bars indicate standard error (SE) of mean $(n=3)$.Cont-Control (distilled water spray), KINKinetin, GB-Glycine betaine. 


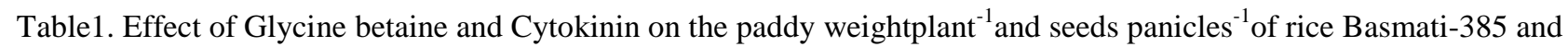
KS-282 under water stress conditions.

\begin{tabular}{|c|c|c|c|c|c|c|}
\hline & \multicolumn{2}{|c|}{ Basmati-385 } & \multicolumn{2}{|l|}{ KS-282 } & \multicolumn{2}{|c|}{ Treatment Mean } \\
\hline Treatment & Paddy Wt. & Seeds Pan. ${ }^{-1}$ & Paddy Wt. & Seeds Pan. ${ }^{-1}$ & Paddy & Seeds \\
\hline Control & $23 \pm 1.8 \mathrm{c}$ & $97 \pm 11.2 \mathrm{~b}$ & $30 \pm 2.0 \mathrm{a}$ & $81 \pm 4.3 \mathrm{a}$ & 26.5 & 89 \\
\hline Kinetin & $46 \pm 0.6 \mathrm{a}$ & $145 \pm 5.5 \mathrm{a}$ & $55 \pm 1.4 b$ & $101 \pm 0.9 b$ & 50.5 & 123 \\
\hline Glycinebetaine & $39 \pm 1.2 \mathrm{~b}$ & $130 \pm 8.5 \mathrm{a}$ & $44 \pm 2.51 b$ & $96 \pm 1.5 \mathrm{~b}$ & 41.5 & 113 \\
\hline Variety Mean & 36.3 & 124 & 43 & 93 & & \\
\hline
\end{tabular}

Paddy -LSD $(0.05)=4.5$ for Basmati-385, LSD $(0.05)=14.50$ for KS-282

Seeds-LSD $(0.05)=30$ for Basmati-385, LSD $(0.05)=12.8$ for KS-282.

Proline accumulation in plant cells plays a crucial role in maintaining osmotic adjustment of the plant under water stress (Ullah et al., 2012). In monocots increase in proline has already been reported with exogenous GB application (Farooq et al., 2008, 2010; Lin Xin et al., 2009). Exogenous GB application at reproductive stage was found to be more effective for proline accumulation as compared to vegetative stage of water stressed sunflower (Iqbal et al., 2011). Similarly, increase in metabolites, particularly amino acids (proline, $\gamma$ aminobutyric acid, alanine, and glycine) and carbohydrates was reported in creeping bent grass with increase in endogenous KIN (Merewitz et al., 2012). KINs foliar spray has been found to increase proline in the leaves of Vigna unguiculata under water stress (Agarwal and Gupta, 1995). Spray of GB and KIN has already been reported to counter the physiological limitations by enhancing the production of proline in many crops under water stress (Zulkarnain et al., 2013; Cha-umet al., 2013). GB might have ameliorated the inhibitory effects of water stress, by stimulating osmotic adjustment of plants (Ullah et al., 2012). Decline in endogenous KIN biosynthesis, content, translocation, and its activity due to water stress might have enhanced with exogenous KIN application, which has been found to be an important factor regulating plant responses to environmental stress (Zulkarnain et al., 2013; Kudoyarova et al., 2006). Further, KIN application might have helped in mobilizing carbohydrates from leaves to the panicles and hence have increased their concentrations in the developing fertilized grain (Merewitz et al., 2012).

Sugars accumulation in response to drought stress is well documented (Mostajeran and RahimiEichi, 2009). Soluble sugars may also act as osmoprotectant, stabilizing cellular membranes and maintaining turgor pressure. Soluble sugars and starch act as substrates in biosynthesis processes and provide energy. They are stored as starch during periods of active photosynthesis, excess carbohydrates in the leaves for subsequent remobilization to other developing tissue (Basu and Minhas, 1991; Lorenzen and Ewing, 1992). GB and KIN play important role in controlling various processes during the plant life cycle. They improve photosynthesis efficiency of plants by affecting enzymes involved in photosynthesis, leaf area, light capture and enhance the efficacy of nutrients. As a result they increase the source capability and redistribute the assimilates and increase the sink strength (Khan et al., 2007). In the present study, higher sugar and starch fillings of the flag leaves from the treated plants caused a noteworthy increase in the grain florets, sugar \&starch quantity under water stressed conditions. This might be due to involvement of KIN in enhancing levels of invertase which has a role in the movement of sugars into the developing reproductive organs of the plant.

Yield of rice crop is a combination of genetic makeup, physiological processes and agronomic attributes and any degree of imbalance in the said parameters may hinder the crop yield. Moreover, availability of sufficient water supply is inevitable to ensure maximum crop harvest in rice being a water loving crop (Basuet al., 2010). In the present study all the yield components adversely affected by drought were ameliorated by foliar spray of GB and KIN. In rice, nearly $80 \%$ of the total carbohydrate gathered in grains is produced by the top two leaves (Gladun and Karpov, 1993). Water stress causes inhibition of assimilate and its movement towards the developing reproductive organs, which might be the reason for the observed decrease in paddy yield plant-1. These adverse effects of water stress were ameliorated in the rice cultivars mainly by the application of GB and KIN through rejuvenation of the sink potential and enhancement of the duration or rate of dry mass accumulation in developing reproductive organs (Davies, 2010). GB and CK could be regarded as essential regulators in the translocation and partitioning of photo assimilates for gain filling in 
rice. Under water stresses $\mathrm{CK}$ increased sink capacity by increasing cell division and influenced source-sink interactions which resulted in the improvement of yield and grain quality of rice (Peleg et al., 2011; Reguera et al., 2013).

\section{Conclusions}

It can be concluded from the above research study that foliar spray of glycine betaine $(100 \mathrm{mM})$ and kinetin $(1 \mathrm{mM})$ in rice affected plant metabolic and yield attributes. These treatments also altered the source / sink equilibrium during water-deficit conditions. Overall treated plants performed better under water-scarcity and yielded more than untreated plants. These bio-regulators could be effectively used for the drought stress amelioration of rice plants, especially during the water stress sensitive reproductive growth stages.

\section{Author contributions}

J. D. made a major contribution in paper writing and overall planning of the study. S. U. K. supervised the research and reviewed the paper. A. $\mathrm{K}$. and S. N. were involved in biochemical analysis and data collection of the research study conducted.

\section{References}

Agarwal, R. K. and S. C. Gupta. 1995. Plant growth substances as osmoregulants under salt stress in callus cultures of cow pea. Ind. J. Plant Physiol. 38:325-327.

Ahadiyat, Y. R. P., Hidayat and U. Susanto. 2014. Drought tolerance, phosphorus efficiency and yield characters of upland rice lines. Emir. J. Food Agric. 26 (1):25-34.

Akram, H. M., A. Ali, A. Sattar, H. S. U. Rehman and A. Bibi. 2013. Impact of water deficit stress on various physiological and agronomic traits of three basmati rice (Oryza sativa L.) cultivars. J. Anim. Plant Sci. 23(5):14151423.

Ashraf, M and M. R. Foolad. 2007. Roles of glycine betaine and proline in improving plant abiotic stress resistance. Environ. Exp. Bot. 59:206-216.

Basu, P. S and J. S. Minhas. 1991. Heat tolerance and assimilate transport in different potato genotype. J. Exp. Bot. 42:861-866.

Basu, S., A. Roychoudhury, P. P. Saha and D. Sengupta. 2010. Differential antioxidative responses of indica rice cultivars to drought stress. Plant Growth Regul. 60:51-59.

Bouman, B. A. M., S. Peng, A. R. Castaòeda and R.
M. Visperas. 2005. Yield and water use of tropical aerobic rice systems. Agric. Water Manag. 74(2):87-105.

Cha-um, S., T. Samphumphuang and C. Kirdmanee. 2013. Glycinebetaine alleviates water deficit stress in indicarice using proline accumulation, photosynthetic efficiencies, growth performances and yield attributes. AJCS. 7(2):213-218.

Cha-um, S., S. Yooyongwechand and K. Supaibulwatana. 2010. Water deficit stress in the reproductive stage of four indica rice (Oryza sativa L.) genotypes. Pak J Bot. 42:3387-3398

Chen, T. H. H. and N. Murata. 2008. Glycine betaine: an effective protectant against abiotic stress in plants. Trends Plant Sci. 13:499-505.

Davies, P. J. 2010. The plant hormones: their nature, occurrence, and functions. Hormones pp. $1-15$.

DuBois, M., K. Gilles, J. Hamilton, P.Rebers and F. Smith. 1956. Colorimetric method for determination of sugars and related substances. Anal. Chem. 28(3):350-356.

Farooq, M., S. M., A. Basra, A. Wahid, Z. A. Cheema, M. A. Cheema and A. Khaliq. 2008. Physiological role of exogenously applied glycinebetaine to improve drought tolerance in fine grain aromatic rice (Oryza sativa L.). J. Agron. Crop Sci. 194:325-333.

Farooq, M., A. Wahid, D. J. Lee, S. A. Sheema and T. Aziz. 2010. Comparative time course action of the foliar applied glycine betaine, salicylic acid, nitrous oxide, brassinosteroids and spermine in improving drought resistance of rice. J. Agron. Crop Sci. 196:336-345.

Gladun, I. V. and E. A. Karpov. 1993. Production and partitioning of assimilates between the panicle and vegetative organs of rice after flowering. Russ. J. Plant Physiol. 40:728-773.

Iqbal, N., Y. Ashraf and M Ashraf. 2011. Modulation of endogenous levels of some key organic metabolites by exogenous application of glycine betaine in drought stressed plants of sunflower (Helianthus annuus L.). Plant Growth Regul. 63:7-12.

IRRI, 1976. Laboratory Manual for Physiological Studies of Rice. 3rdEd. The IRRI. Los Banos, the Phillipines. 
Khan, N. A., S. Singh, R. Nazar and P. M. Lone. 2007. The source sinks relationship in mustard. Asian Aust. J. Plant. Sci. Biotechnol.1:10-18.

Kudoyarova, G. R., L. B. Vysotskaya, A. Cherkozyanova and I. C. Dodd. 2006. Effects of partial root zone drying on the concentration of zeatin-type cytokinins in tomato (Solanum lycopericum L.) xylem sap and leaves. J. Exp. Bot. 58:161-168.

Laborte, A. G., C. A. J. M. de Bie, E. M. A. Smaling, P. F. Moya, A. A. Boling and M. K. van Ittersum. 2012. Rice yields and yield gaps in South East Asia: Past trends and future outlook. Eur. J. Agron. 36:9-20.

Li Xin, Z., L. Sheng Xiu and L. Zong Suo. 2009. Differential plant growth and osmotic of two maize (Zea mays L.) cultivars to exogenous glycinebetaine application under drought stress. Plant Growth Regul. 58:297-305.

Lorenzen, J. H. and E. Ewing. 1992. Starch accumulation in leaves of potato (Solanum tuberosum L.) during the first 18 days of photoperiod treatment. Ann. Bot. 69:481-485.

Ma, X. L, Y. J.Wang, S. L. Xie, C. Wang, and W. Wang. 2007. Glycinebetaine application ameliorates negative effects of drought stress in tobacco. Russ. J. Plant Physiol. 54:534-541.

Merewitz, E. B., H. Du and B. Huang. 2012. Elevated cytokinin content in ipt transgenic creeping bent grass promotes drought tolerance through regulating metabolite accumulation. J. Exp. Bot. 63(3):1315-1328.

Mostajeran, A. and V. Rahimi-Eichi. 2009. Effects of drought stress on growth and yield of rice (Oryza sativa L.) cultivars and accumulation of proline and soluble sugars in sheath and blades of their different ages leaves. American-Eurasian J. Agric. Environ. Sci. 5(2):264-272.
Peleg, Z., M. Reguera, E. Tumimbang, H. Walia and E. Blumwald. 2011. Cytokinin-mediated source/sink modifications improve drought tolerance and increase grain yield in rice under water-stress. Plant Biotechnol. J. 1-12.

Radchuk, V., R. Radchuk, Y. Pirko, R. Vankova, A. Gaudinova, V. Korkhovoy, A. Yemets, H. Weber, W.Weschke and Y. B. Blume. 2012. A somaclonalline SE7 of finger millet (Eleusine coracana) exhibits modified cytokinin homeostasis and increased grain yield. J. Exp. Bot. 63(15):5497-5506.

Reguera, M., Z. Peleg,Y. M. Abdel-Tawab, B. T. Ellen, A. Carla, B. Delatorre, A. Carla A. Delatorre and E. Blumwald. 2013. Stressinduced cytokinin synthesis increases drought tolerance through the coordinated regulation of carbon and nitrogen assimilation in rice. Plant Physiol. 163:1609-1622.

Rezaei, M. A., I. Jokar, M. Ghorbanli, B. Kaviani and A. Kharabian-Masouleh. 2012. Morphophysiological improving effects of exogenous glycine betaine on tomato (Lycopersicum esculentum Mill.) cv. PS under drought stress conditions. Plant Omics J. 5:79-86.

Thapa, G., M. Dey, L. Sahoo and S. K.Panda. 2011. An insight into the drought stress induced alterations in plants. Biol. Plant. 55:603-613.

Ullah, F., A. Bano and A. Nosheen, 2012. Effects of plant growth regulators on growth and oil quality of canola (Brassica napus L.) under drought stress. Pak. J. Bot. 44(6):1873-1880. 